Received: 21.12 .2020

Revised: 29.01 .2021

Accepted: 26.02 .2021

DOI: $10.17804 / 2410-9908.2021 .1 .026-037$

\title{
MODEL REPRESENTATION OF AN AXISYMMETRIC STEEL-ALUMINUM SAMPLE FOR SIMULATION OF A SEPARATION TEST
}

\author{
D. I. Kryuchkov ${ }^{\text {a)* }}$, A. G. Zalazinsky ${ }^{\text {) }}$ \\ Institute of Engineering Science, Ural Branch of the Russian Academy of Sciences, \\ 34, Komsomolskaya St., Ekaterinburg, 620049, Russian Federation \\ a) (iD http://orcid.org/0000-0001-8585-3544; ख kru4koff@bk.ru; \\ b) (iD http://orcid.org/0000-0001-8352-5475; ख zalaz@list.ru \\ *Corresponding author. E-mail: kru4koff@bk.ru \\ Address for correspondence: ul. Komsomolskaya, 34, 620049, Ekaterinburg, Russian Federation \\ Tel.: +7 (343) 37450 51; fax: +7 (343) 3745330
}

The object of research is a bimetallic composite material with a thin intermediate layer of aluminum. The aim of the work is to determine the features of the stress-strain state at the interlayer boundaries of a steel-aluminum composite material with a thin layer using the computational experiment method and to calculate separation resistance using the energy criterion. The stress-strain state along the boundaries of the joint at delamination is determined from the results of modeling the deformation of axisymmetric samples made of a steel-aluminum bimetallic composite material with a thin intermediate layer of aluminum. A series of computational experiments with varying the critical rate of elastic energy release under separation conditions, including under the combined influence of low temperatures and static loads, is implemented. The energy criterion is used to evaluate the stress level that leads to the separation of the bimetallic compound. The dependence of the separation resistance along the ring contour on the critical rate of elastic energy release, which is variable in the range of 0.1 to $0.5 \mathrm{~N} / \mathrm{mm}$, is calculated. It is established that, for the studied variants of the computational experiment, a rigid stress state with a predominance of normal tensile stresses is realized at the place of delamination onset.

Keywords: simulation modeling, deformation, axisymmetric sample, steel-aluminum composite material, joint boundary, crack.

\section{Acknowledgment}

We appreciate the effort of Dr. Berezin, senior professor of the Chair of Information Technologies and Design Automation, UrFU, in making computational experiments.

\section{References}

1. Krueger R. Virtual crack closure technique: History, approach, and applications. Applied Mechanics Reviews, 2004, 57 (2), pp. 109-143. DOI: 10.1115/1.1595677.

2. Valvo P.S. A Physically Consistent Virtual Crack Closure Technique for I/II/III Mixedmode Fracture Problems. Procedia Materials Science, 2014, vol. 3, pp. 1983-1987. DOI: 10.1016/j.mspro.2014.06.319.

3. $\quad$ Liu P.F., Hou S.J., Chu J.K., Hu X.Y., Zhou C.L., Liu Y.L., Zheng J.Y., Zhao A., Yan L. Finite element analysis of post buckling and delamination of composite laminates using virtual crack closure technique. Composite Structures, 2011, vol. 93, iss. 5, pp. 1549-1560. DOI: 10.1016/j.compstruct.2010.12.006. 
4. Xie D., Biggers S. Strain energy release rate calculation for a moving delamination front of arbitrary shape based on the virtual crack closure technique. Part I: Formulation and validation. Engineering Fracture Mechanics, 2006, vol. 73, iss. 6, pp. 771-785. DOI: 10.1016/j.engfracmech.2005.07.013.

5. Perov S.N., Chernyakin S.A. Research the applicability of finite element method for estimation the parameters of fracture mechanics of constructive elements from composites. Izvestiya Samarskogo Nauchnogo Tsentra Rossiyskoy Akademii Nauk, 2013, vol. 15, no. 4 (2), pp. 480-483. (In Russian).

6. Chernyakin S.A., Skvortsov Y.V. Analysis of delamination propagation in composite structures. Vestnik SibGAU, 2014, no. 4 (56), pp. 249-255. (In Russian).

7. Glushkov S.V., Skvortsov Y.V., Perov S.N., Chernyakin S.A. Finite element analysis of panels with surface cracks. AIP Conference Proceedings, 2017, vol. 1798, 020059. DOI: $10.1063 / 1.4972651$.

8. Marjanović M., Meschke G., Vuksanović D. A finite element model for propagating delamination in laminated composite plates based on the Virtual Crack Closure method. Composite Structures, 2016, vol. 150, pp. 8-19. DOI: 10.1016/j.compstruct.2016.04.044.

9. Liu P.F., Islam M.M. A nonlinear cohesive model for mixed-mode delamination of composite laminates. Composite Structures, 2013, vol. 106, pp. 47-56. DOI: 10.1016/j.compstruct.2013.05.049.

10. Harper P.W., Hallett S.R. Cohesive zone length in numerical simulations of composite delamination. Engineering Fracture Mechanics, 2008, vol. 75, pp. 4774-4792. DOI: 10.1016/j.engfracmech.2008.06.004.

11. Azimi M., Mirjavadi S.S., Asli S.A., Hamouda A.M.S. Fracture Analysis of a Special Cracked Lap Shear (CLS) Specimen with Utilization of Virtual Crack Closure Technique (VCCT) by Finite Element Methods. Journal of Failure Analysis and Prevention, 2017, vol. 17, iss. 2, pp. 304-314. DOI: 10.1007/s11668-017-0243-1.

12. Bonhomme J., Argüelles A., Viña J., Viña I. Numerical and experimental validation of computational models for mode I composite fracture failure. Computational Materials Science, 2009, vol. 45, pp. 993-998. DOI: 10.1016/j.commatsci.2009.01.005.

13. Shokrieh M.M., Rajabpour-Shirazi H., Heidari-Rarani M., Haghpanahi M. Simulation of mode I delamination propagation in multidirectional composites with R-curve effects using VCCT method. Computational Materials Science, 2012, vol. 65, pp. 66-73. DOI: 10.1016/j.commatsci.2012.06.025.

14. Martinez X., Rastellini F., Oller S., Floresa F., Oñate E. Computationally optimized formulation for the simulation of composite materials and delamination failures. Composites Part B: Engineering, 2011, vol. 42, iss. 2, pp. 134-144. DOI: 10.1016/j.compositesb.2010.09.013.

15. Skvortsov Yu.V., Chernyakin S.A., Glushkov S.V., Perov S.N. Simulation of fatigue delamination growth in composite laminates under mode I loading. Applied Mathematical Modelling, 2016, vol. 40, pp. 7216-7224. DOI: 10.1016/j.apm.2016.03.019.

16. Amiri-Rad A., Mashayekhi M., van der Meer F.P. Cohesive zone and level set method for simulation of high cycle fatigue delamination in composite materials. Composite Structures, 2017, vol. 160, pp. 61-69. DOI: 10.1016/j.compstruct.2016.10.041.

17. May M., Hallett S.R. An advanced model for initiation and propagation of damage under fatigue loading - part I: Model formulation. Composite Structures, 2011, vol. 93, pp. 2340-2349. DOI: 10.1016/j.compstruct.2011.03.022.

18. Smirnov S.V., Myasnikova M.V., Igumnov A.S. Determination of the local shear strength of a layered metal composite material with a ductile interlayer after thermocycling. Diagnostics, Resource and Mechanics of materials and structures, 2016, iss. 4, pp. 46-56. DOI: 10.17804/24109908.2016.4.046-056.

19. Kachanov L.M. Osnovy mekhaniki razrusheniya [Fundamentals of Fracture Mechanics]. Moscow, Nauka Publ., 1974, 312 p. (In Russian). 
Подана в журнал: 21.12.2020

УДК 539.415

DOI: $10.17804 / 2410-9908.2021 .1 .026-037$

\title{
МОДЕЛЬНОЕ ПРЕДСТАВЛЕНИЕ СТАЛЕАЛЮМИНИЕВОГО ОСЕСИММЕТРИЧНОГО ОБРАЗЦА ДЛЯ ИМИТАЦИОННОГО МОДЕЛИРОВАНИЯ ИСПЫТАНИЯ НА ОТРЫВ
}

\author{
Д. И. Крючков ${ }^{\text {a)* }}$, А. Г. Залазинский ${ }^{\text {) }}$ \\ Институт машиноведения (ИМАШ) УрО РАН, \\ 34, ул. Комсомольская, г. Екатеринбург, Российская Федерация \\ a) iD http://orcid.org/0000-0001-8585-3544; ख kru4koff@bk.ru; \\ б) (iD http://orcid.org/0000-0001-8352-5475; zalaz@list.ru \\ *Ответственный автор. Эл. почта: kru4koff@bk.ru \\ Адрес для переписки: ул. Комсомольская, 34, 620049, г. Екатеринбург, Российская Федерация \\ Тел.: +7 (343) 374-50-51; факс: +7 (343) 374-53-30
}

Объектом исследования является модель биметаллического композитного материала с тонким промежуточным слоем из алюминия. Цель работы - с использованием метода вычислительного эксперимента определить особенности напряженно-деформированного состояния на межслойных границах сталеалюминиевого композитного материла с тонкой прослойкой, и рассчитать сопротивление отрыва с использованием энергетического критерия. По результатам моделирования процесса деформирования осесимметричных образцов из сталеалюминиевого биметаллического композитного материала с тонким промежуточным слоем алюминия определено напряженно-деформированное состояние по границам соединения, при котором происходит расслоение. Реализована серия вычислительных экспериментов с варьированием критической скорости высвобождения упругой энергии в условиях отрыва, в том числе в условиях совместного влияния низких температур и статических нагрузок. С использованием энергетического критерия оценивается уровень напряжений, приводящий к расслоению биметаллического соединения. Рассчитана зависимость сопротивления отрыву по кольцевому контуру от величины критической скорости высвобождения упругой энергии, варьируемой в диапазоне 0,1-0,5 Н/мм. Установлено, что для исследованных вариантов вычислительного эксперимента в месте начала расслоения реализуется жесткое напряженное состояние с преобладанием нормальных растягивающих напряжений.

Ключевые слова: имитационное моделирование, деформирование, осесимметричный образец, сталеалюминиевый композитный материал, граница соединения, трещина.

\section{1. Введение}

В настоящее время слоистые металлические композитные материалы (СМКМ) на основе низкоуглеродистых сталей и алюминиевых сплавов, полученные методом горячей прокатки, находят все большее применение. При разработке процессов и изготовлении многослойных металлов оценку качества соединения производят путем механических и технологических испытаний образцов композиций, а также металлографических исследований границ соединения их компонентов. Однако известные способы механических испытаний, технологические пробы и металлографические исследования не всегда позволяют оценить качество соединения слоев, в частности для композитов с толщиной слоев или прослойкой $<1$ мм.

Одним из типовых видов повреждения слоистых материалов при деформации является расслоение по границе соединения. Поэтому определение прочностных характеристик границ раздела слоев композита при отрыве и сдвиге представляют практическую ценность 
для обеспечения надежности изделия в условиях эксплуатации. Одним из распространенных способов определения прочности соединения слоев биметаллических соединений является испытание на срез и на отрыв с определением сопротивления срезу и отрыву по плоскости соприкосновения слоев. К нормативным документам исследования биметаллических слоистых материалов относятся технические условия ТУ 27.32.09.010-05. Согласно ТУ регламентированы образцы для испытаний. Однако для многослойных материалов оценка прочностных характеристик зависит от многих факторов, таких как схема испытания, конфигурация и размеры образца. Для одних и тех же условий испытаний различное количество слоев и схемы чередования слоев, а также их толщина, накладывают свои особенности формоизменения рассматриваемых материалов. Поэтому выявление закономерностей разрушения на границах многослойных металлических материалов при различных видах воздействия представляет собой достаточно сложную задачу, решение которой по-прежнему актуально.

Для описания процессов возникновения и роста трещин, а также расслоения, в СМКМ обычной практикой стало применение подходов механики разрушения с использованием современных методов вычислений. Для изучения поведения композитных структур и определения условий инициализации и роста расслоения находят применение подходы, основанные на расчете удельной работы расслоения. Одним из таких подходов является метод виртуального закрытия трещин (VCCT) [1]. Обоснование метода дано в работе [2]. Одно из главных допущений VCCT сводится к тому, что рост трещин происходит по заранее определенному пути, а именно по межфазным границам. В настоящее время этот подход применяется с использованием имитационного моделирования методом конечных элементов [3-9]. В работах [10-14] даны рекомендации по численной реализации подхода, установлены ограничения применения существующих формул, используемых для прогнозирования численной длины когезионной зоны, и демонстрируются модификации метода, необходимые для повышения точности, дано разъяснение относительно минимального количества конечных элементов в пределах зоны когезионного взаимодействия. Метод VCCT также успешно применяют в модифицированном виде для прогнозирования усталостного многоциклового разрушения конструкционных материалов [15-17].

Цель работы - с использованием метода вычислительного эксперимента определить особенности напряженно-деформированного состояния на межслойных границах сталеалюминиевого композитного материла с тонкой прослойкой, и рассчитать сопротивление отрыва с использованием энергетического критерия.

\section{2. Материал и методика}

В вычислительных экспериментах для изучения процессов формоизменения в слоистых композитах применялась модель образца, представленная на рис. 1.

Образец представляет собой осесимметричное тело, закрепленное на опоре, состоящее из трех пластин с вырезом под пуансон. Толщину слоев 1,2 и 3 приняли следующими: 7,2 мм; 0,6 и 7,2 мм соответственно. В слоях 1 и 2 выполнено цилиндрическое отверстие под пуансон 4. Размер выреза под пуансон варьировался; его диаметр $d$ составлял 6, 10 и 16 мм. Диаметр нижней пластины оставался постоянным $D=20$ мм. Таким образом, отношение $l / D$ изменялось в диапазоне 0,1-0,35. Имитационное моделирование проводили методом конечных элементов. Моделирование процесса осуществляли с применением расчетного модуля Standard в рамках CAE-системы Abaqus. При этом была определена исходная трещина: $l_{\text {тр }}^{0}=0,4$ мм. Для реализации деформации образца задавали перемещение пуансона 4 и фиксировали усилие $P$. Для каждого слоя в табличной форме задавали свойства упругопластической модели материала с нелинейным упрочнением. Модель материала для верхней пластины соответствует свойствам стали $12 \mathrm{X} 18 \mathrm{H} 10 \mathrm{~T}$; для промежуточного слоя задавали свойства алюминиевого сплава АД1; для нижней пластины приняты свойства сплава АМг6. Свойства материалов заимствовали из работы [18], кривые упрочнения показаны на рис. 2. Модуль 
упругости $E$ и коэффициент Пуассона $v$ взяты также из литературы. Инструмент задавался как абсолютно жесткий. Контактное взаимодействие между заготовкой и инструментом описывалось законом трения Амантона-Кулона, коэффициент трения $\mu=0,2$.

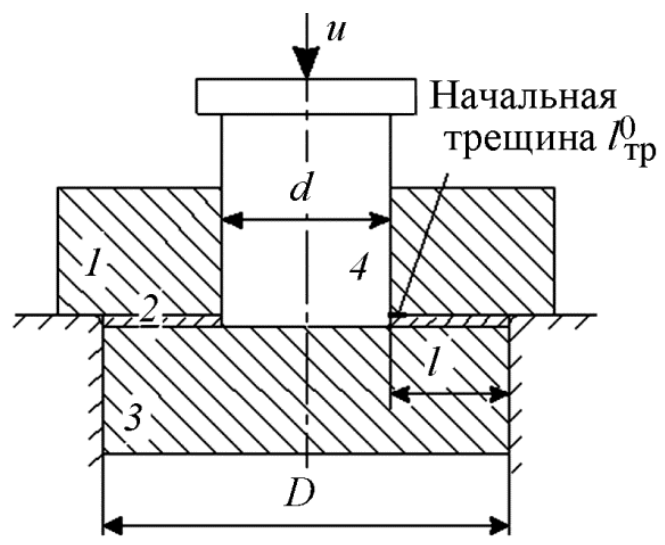

Рис. 1. Геометрическая модель образца: 1 - пластина из стали 12 Х18Н10Т; 2 - промежуточный слой сплава АД1; 3 - пластина из сплава АМг6; 4 - пуансон

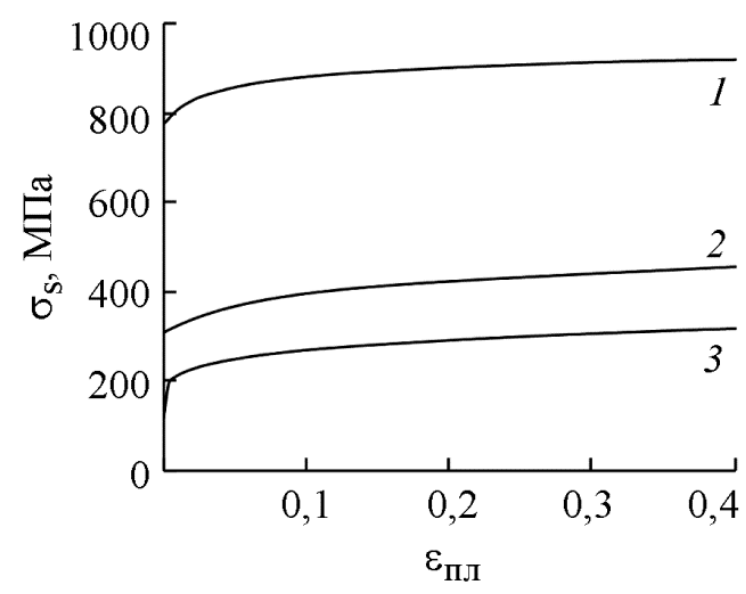

Рис. 2. Кривые упрочнения материалов компонентов композита:

$$
1 \text { - 12Х18Н10Т; } 2 \text { - АМг6; } 3 \text { - АД1 }
$$

Напряжения $\sigma$ рассчитывали как отношение значения прилагаемой на пуансон нагрузки $P$ к площади контакта на межслойной границе по формуле:

$$
\sigma=P /\left(\pi\left((\mathrm{D} / 2)^{2}-\left(\left(d / 2+l_{\mathrm{Tp}}\right)^{2}\right)\right)\right.
$$

где $P$ - значение усилия на пуансон; $l_{\text {тр }}$ - длина трещины; $D, d$ - параметры геометрической модели образца.

Для определения условий инициализации процесса образования и роста расслоения применяли критерий, обоснованный энергетическим условием [3], в соответствии с которым рассчитанная в узле скорость высвобождения упругой энергии $G_{I}$ в условиях отрыва должна быть равна или выше значения критической скорости высвобождения упругой энергии $G_{I C}$, заданной пользователем. Расчет энергетического условия осуществляется в Abaqus по формуле:

$$
f=G_{I} / G_{I C} \geq 1 \text {. }
$$


Значение $G_{I}$ вычисляется следующим образом:

$$
G_{I}=-R_{2} \Delta v /(2 \Delta a b)
$$

где $R_{2}$ - реакция в вершине трещины, препятствующая ее раскрытию; $\Delta v$ - вертикальное смещение между ближайшими к вершине трещины узлами в локальных координатах; $b$ - ширина; $\Delta a$ - длина элемента на фронте трещины (рис. 3).

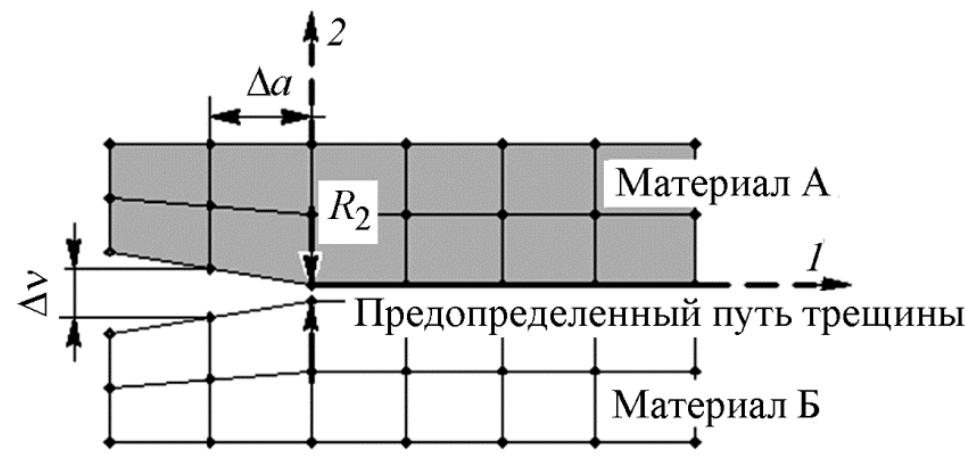

Рис. 3. Представление параметров расчета высвобождения упругой энергии $G_{I}$ при расслоении образца по межслойной границе

\section{3. Результаты и их обсуждение}

Сравнительный анализ напряженно-деформированного состояния проводили при варьировании $G_{I C}$ в диапазоне $0,1-0,5 \mathrm{H} /$ мм. Напряженно-деформированное состояние определяли по границам соединения с прослойкой для момента, при котором инициируется расслоение. Распределение компонентов тензора напряжений и пластических деформаций по границе соединения оценивали визуально, пример представлен на рис. 4.

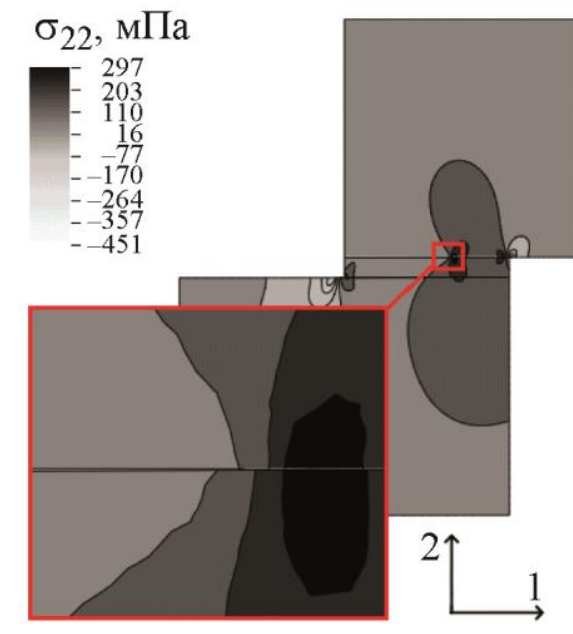

$a$

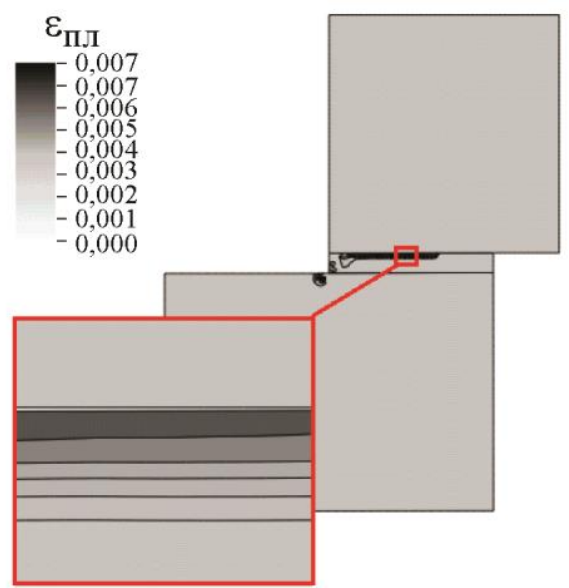

$\sigma$

Рис. 4. Распределение нормальных напряжений $\sigma_{22}(a)$ и пластических деформаций $\varepsilon_{\text {пл }}(б)$ по границе соединения в момент, предшествующий самопроизвольному распространению трещины: $l / D=0,2 ; G_{I C}=0,2 \mathrm{H} / \mathrm{Mm}$

Поле распределения пластической деформации (рис. 4 б) по границе соединения характеризуется тем, что любому, в том числе хрупкому разрушению металлов и сплавов предшествует локализация пластического течения в тонком слое у вершины трещины [19]. 
Показано, что пластически деформируется только материал промежуточного слоя (алюминий АД1), а пластическая зона имеет простой профиль и расположена на границе соединения. В то же время степень пластической деформации в момент, предшествующий самопроизвольному распространению трещины, зависит от конфигурации образца.

При конечно-элементном расчете фиксировалась нагрузка на пуансон $P$ в зависимости от перемещения пуансона $u_{\text {п }}$ (рис. 5) для расчета напряжения $\sigma$ по формуле (1). Пики на графике соответствуют началу раскрытия трещины.

Установлено, что минимальные значения пластической деформации в слое наблюдаются при конфигурации образца с соотношением $l / D=0,35$. Для каждой конфигурации образца наблюдается линейная зависимость степени пластической деформации $\varepsilon_{\text {пл }}$ от критической скорости высвобождения упругой энергии $G_{I C}$ в условиях отрыва.

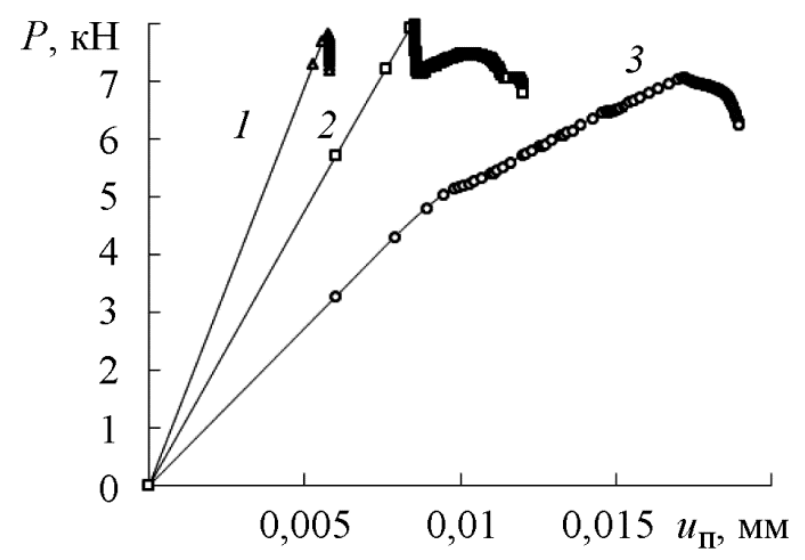

Рис. 5. Зависимость усилия $P$ от перемещения пуансона $u_{\Pi}: G_{I C}=0,1 \mathrm{H} / \mathrm{мm:}$

$$
1-l / D=0,1 ; 2-l / D=0,2 ; 3-l / D=0,35
$$

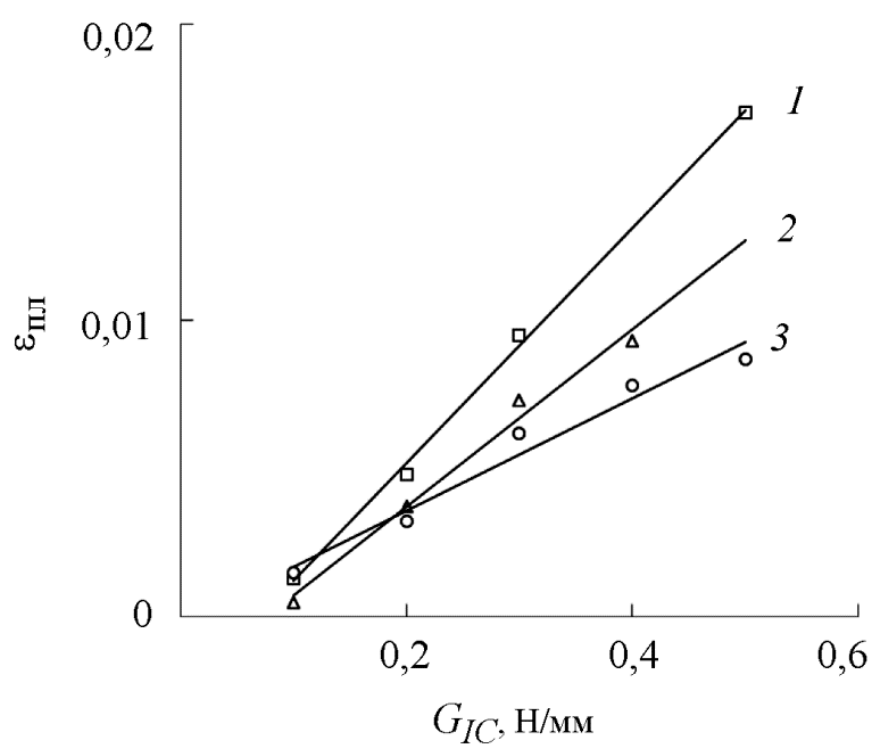

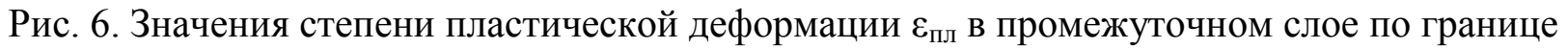
соединения в момент, предшествующий самопроизвольному распространению трещины:

$$
1-l / D=0,1 ; 2-l / D=0,2 ; 3-l / D=0,35
$$

В исследуемом образце интенсивная пластическая деформация локализуется в промежуточном слое вблизи пуансона. В зависимости от используемого в расчетах значений $G_{I C}$ до момента отрыва в прослойке степень пластической деформации составляет 0,0003 до 0,02 соответствен- 
но (рис. 6). Для всех вариантов расчета в месте инициирования расслоения показатель напряженного состояния $k=0,58$, а показатель Лоде-Надаи $\mu_{\sigma}=+1$. Эти величины практически не меняются на протяжении процесса деформирования. Такие показатели характеризуют жесткое напряженное состояние с преобладанием нормальных растягивающих напряжений.

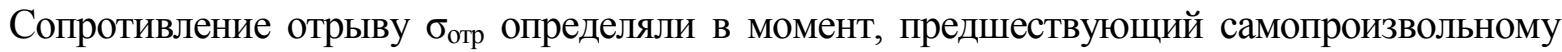
распространению трещины, по диаграмме зависимости напряжения б от перемещения пуансона

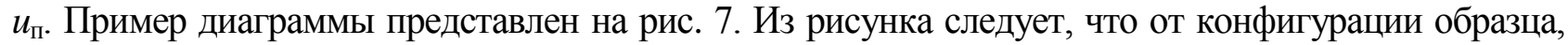
т. е. соотношения $l / D$, значение сопротивления отрыву практически не зависит.

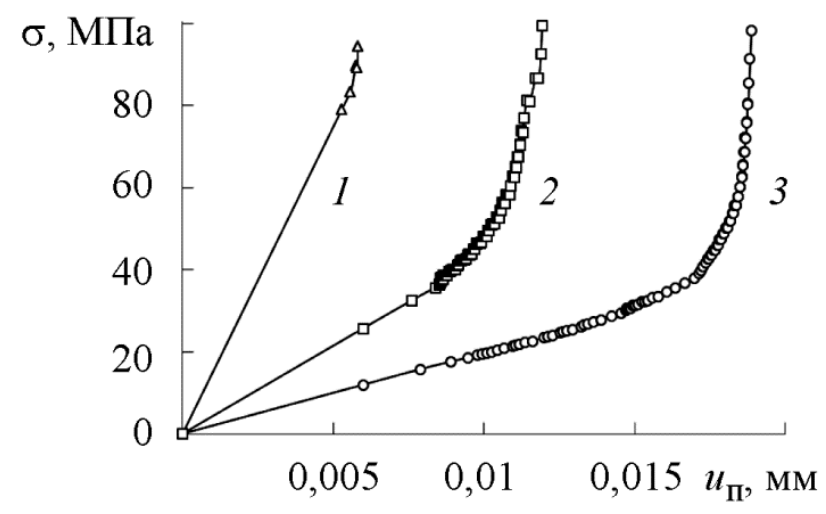

Рис. 7. Зависимость напряжения $\sigma$ от перемещения пуансона $u_{\Pi}$ при $G_{I C}=0,1 \mathrm{H} / \mathrm{Mm:}$

$$
1-l / D=0,1 ; 2-l / D=0,2 ; 3-l / D=0,35
$$

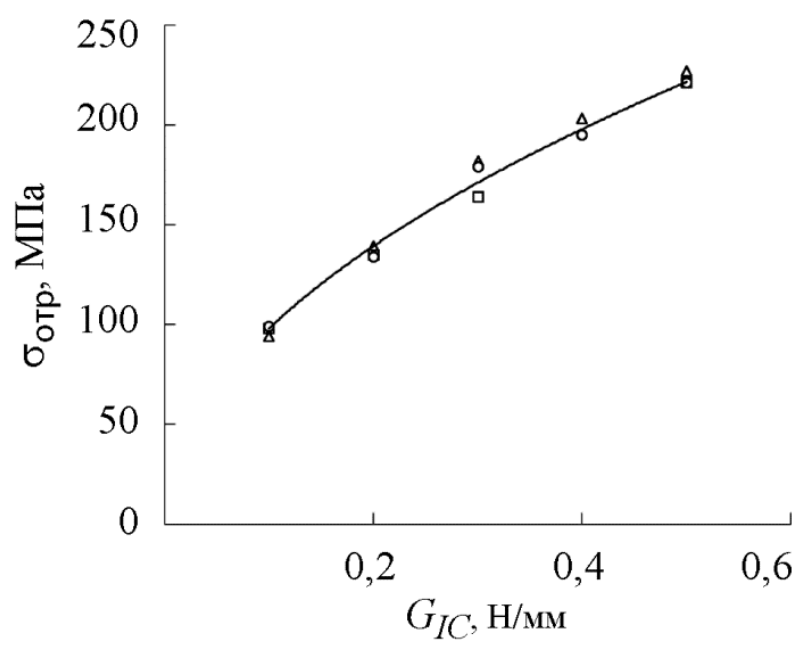

Рис. 8. Зависимость сопротивления отрыву $\sigma_{\text {отр }}$ от величины критической скорости высвобождения упругой энергии $G_{I C}: \Delta-l / D=0,1 ; \square-l / D=0,2 ; \circ-l / D=0,35$

По результатам вычислительных экспериментов построена зависимость сопротивле-

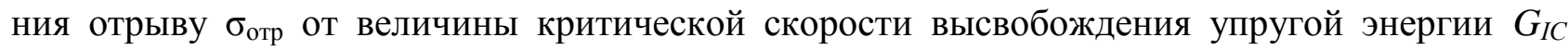
в условиях отрыва (рис. 8). Определены коэффициенты уравнения, аппроксимирующего экспериментальные данные в интервале $G_{I C}=[0,1 ; 0,5]$ :

$$
\sigma_{\text {отр }}=314,56\left(G_{I C}\right)^{0,5062} \text {. }
$$

Коэффициент детерминации $R^{2}=0,9907$. 


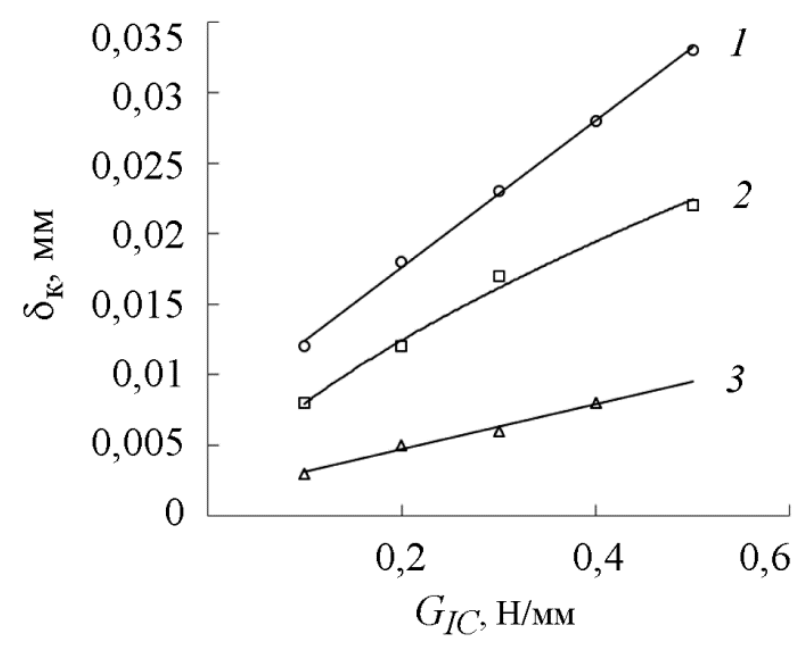

Рис. 9. Зависимость критического раскрытия трещины $\delta_{\text {к }}$ от величины критической скорости высвобождения упругой энергии $G_{I C}$ при отрыве

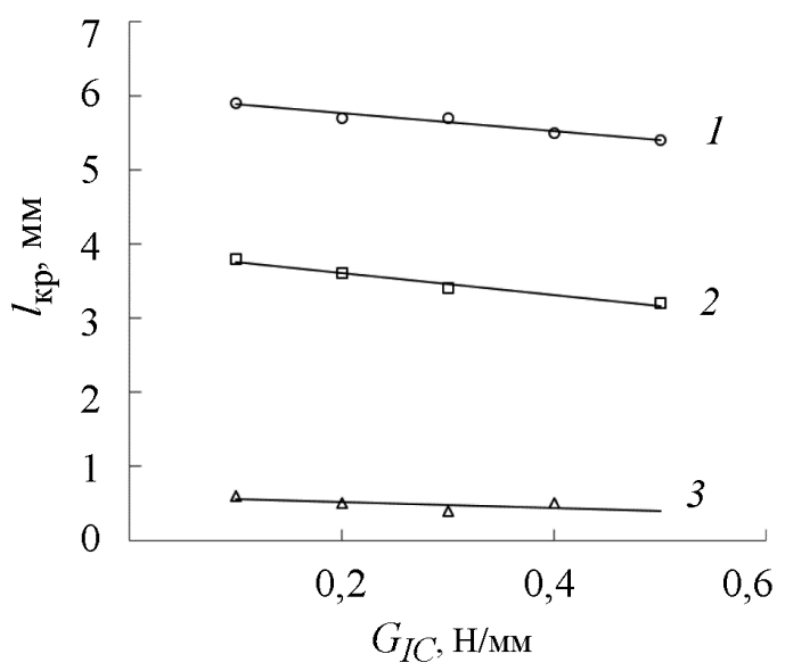

Рис. 10. Зависимость критической длины трещины $l_{\text {кр }}$ от величины критической скорости высвобождения упругой энергии $G_{I C}$ при отрыве

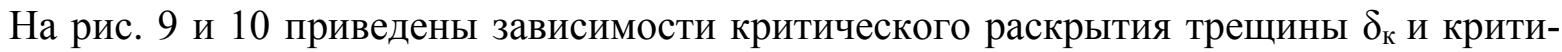
ческой длины трещины $l_{\text {кр }}$ от величины $G_{I C}$. Зависимости имеют линейный характер. Для каждой конфигурации образца с увеличением $G_{I C}$ характерен рост значения критического

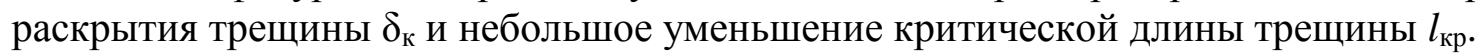

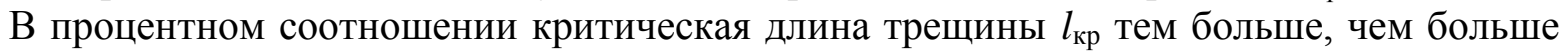
параметр конфигурации образца $l$ (рис. 11). При $l / D=0,1$ это соотношение 25-30 \%, а при

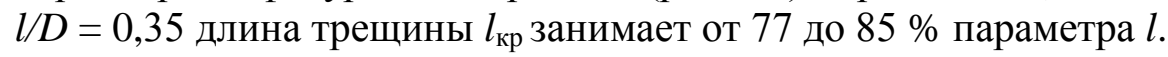

Поскольку при низких температурах сопротивление пластической деформации (предел текучести, прочности, твердости), как правило, возрастает, а пластичность и вязкость с понижением температуры обычно уменьшаются, то для учета влияния низких температур были изменены параметры кривых упрочнения материалов. Пластические свойства материалов были взяты для температуры $-50{ }^{\circ} \mathrm{C}$. После изменения пластических свойств всех слоев образца был проведен расчет и построена зависимость критической скорости высвобождения упругой энергии $G_{I C}$ в условиях отрыва от сопротивления отрыву $\sigma_{\text {отр }}$ (рис. 12 ).

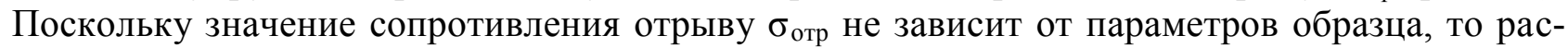
чет проводился на одной конфигурации при $l / D=0,35$. Зависимость показывает, что при 
воздействии отрицательных температур на образец для одного и того же значения $G_{I C}$ сопротивление отрыва ниже.

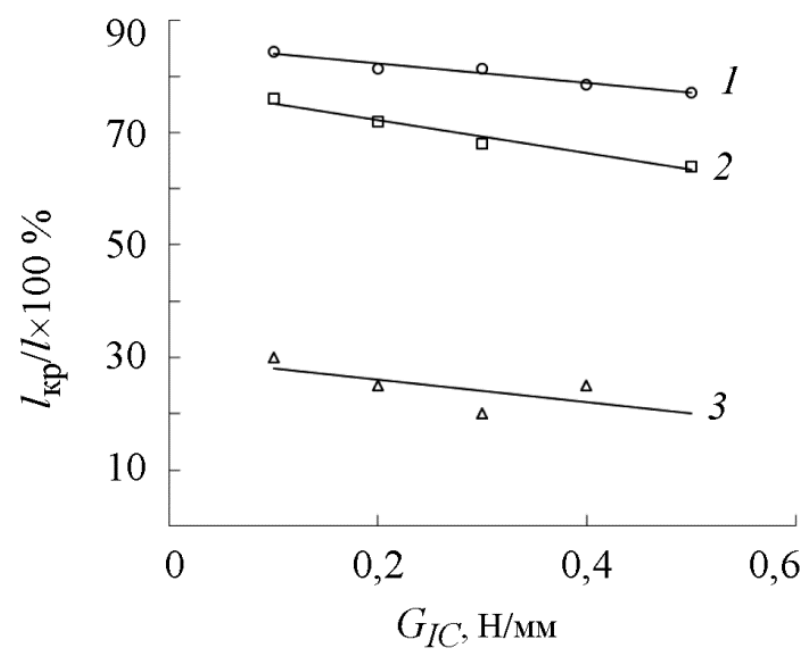

Рис. 11. Соотношение критической длины трещины $l_{\text {кр }}$ в зависимости от параметра конфигурации образца $l / D$ и величины критической скорости высвобождения упругой энергии $G_{I C}$ для отрыва

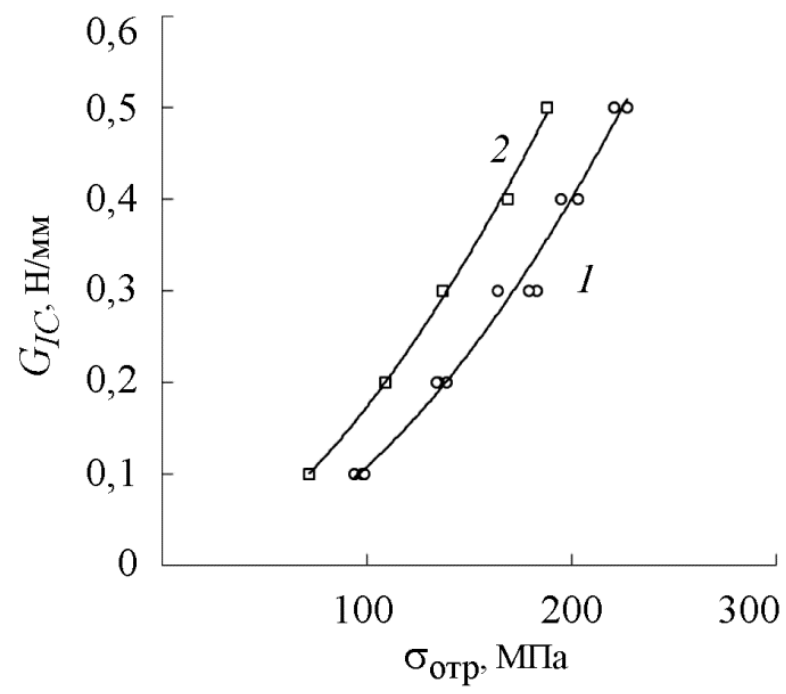

Рис. 12. Критическая скорость высвобождения упругой энергии $G_{I C}$ в условиях отрыва в зависимости от сопротивления отрыву $\sigma_{\text {отр }}: 1-$ для образцов при $T=20{ }^{\circ} \mathrm{C}$;

$$
2 \text { - для образцов при } T=-50{ }^{\circ} \mathrm{C}
$$

Для определения критической скорости высвобождения упругой энергии $G_{I C}$ приведены аппроксимирующие уравнения (5) и (6):

для образцов при $T=20^{\circ} \mathrm{C}$

$$
G_{I C}=\left(2 \times 10^{-5}\right)^{*}\left(\sigma_{\text {отр }} \mid 20\right)^{1,8919}
$$

для образцов при $T=-50{ }^{\circ} \mathrm{C}$

$$
G_{I C}=\left(8 \times 10^{-5}\right) \times\left(\sigma_{\text {отр }} \mid-50\right)^{1,6587}
$$


Для оценки относительной погрешности при аппроксимации функций (5) и (6) рассчитан коэффициент детерминации $R^{2}$, равный 0,99 и 0,9987 соответственно.

После проведения натурного эксперимента по расслоению и определения реального

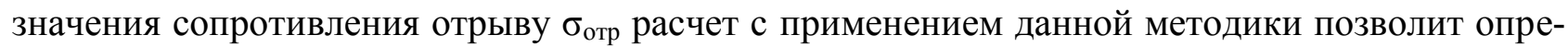
делить значения $G_{I C}$ и использовать его при моделировании реальной конструкции на прочность.

\section{4. Заключение}

С целью исследования процессов формоизменения в слоистых металлических композитах и прогнозирования их расслоения при механическом воздействии проведена серия вычислительных экспериментов. Показано, что инициализация и рост трещины происходит на границе алюминиевой прослойки в области с преобладанием нормальных растягивающих напряжений. Несмотря на то, что подход реализует модель хрупкого разрушения, в прослойке в начальной стадии пластического течения возникают и развиваются пластические деформации. Этот факт необходимо учитывать при разработке методики механических испытаний.

\section{Благодарность}

Выражаем благодарность в проведении вычислительных экспериментов старшему преподавателю кафедры информационных технологий и автоматизации проектирования УрФУ к.т.н. И.М. Березину.

\section{Литература}

1. Krueger R. Virtual crack closure technique: History, approach, and applications // Applied Mechanics Reviews. - 2004. - 57 (2). - P. 109-143. - DOI: 10.1115/1.1595677.

2. Valvo P. S. A Physically Consistent Virtual Crack Closure Technique for I/II/III Mixedmode Fracture Problems // Procedia Materials Science. - 2014. - Vol. 3. - P. 1983-1987. DOI: $10.1016 /$ j.mspro.2014.06.319.

3. Finite element analysis of post buckling and delamination of composite laminates using virtual crack closure technique / P. F. Liu, S. J. Hou, J. K Chu., X. Y. Hu, C. L. Zhou, Y. L. Liu, J. Y. Zheng, A. Zhao, L. Yan // Composite Structures. - 2011. - Vol. 93, iss. 5. - P. 1549-1560. DOI: 10.1016/j.compstruct.2010.12.006.

4. Xie D., Biggers S. Strain energy release rate calculation for a moving delamination front of arbitrary shape based on the virtual crack closure technique. Part I: Formulation and validation // Engineering Fracture Mechanics. - 2006. - Vol. 73, iss. 6. - P. 771-785. DOI: 10.1016/j.engfracmech.2005.07.013.

5. Перов С. Н., Чернякин С. А. Исследование применимости метода конечных элементов для оценки параметров механики разрушения конструктивных элементов из композиционных материалов // Известия Самарского научного центра Российской академии наук. 2013. - Т. 15, № 4 (2). - С. 480-483.

6. Чернякин С. А., Скворцов Ю. В. Анализ роста расслоений в композитных конструкциях // Вестник Сибирского государственного аэрокосмического университета им. академика М. Ф. Решетнева. - 2014. - № 4 (56). - С. 249-255.

7. Finite element analysis of panels with surface cracks / S. V. Glushkov, Y. V. Skvortsov, S. N. Perov, S. A. Chernyakin // AIP Conference Proceedings. - 2017. - 1798. - P. 020059. DOI: $10.1063 / 1.4972651$.

8. Marjanović M., Meschke G., Vuksanović D. A finite element model for propagating delamination in laminated composite plates based on the Virtual Crack Closure method // Composite Structures. - 2016. - Vol. 150. - P. 8-19. - DOI: 10.1016/j.compstruct.2016.04.044. 
9. Liu P. F., Islam M. M. A nonlinear cohesive model for mixed-mode delamination of composite laminates // Composite Structures. - 2013. - Vol. 106. - P. 47-56. DOI: $10.1016 /$ j.compstruct.2013.05.049.

10. Harper P. W., Hallett S. R. Cohesive zone length in numerical simulations of composite delamination // Engineering Fracture Mechanics. - 2008. - Vol. 75. - P. 4774-4792. DOI: 10.1016/j.engfracmech.2008.06.004.

11. Fracture Analysis of a Special Cracked Lap Shear (CLS) Specimen with Utilization of Virtual Crack Closure Technique (VCCT) by Finite Element Methods / M. Azimi, S. S. Mirjavadi, S. A. Asli, A. M. S. Hamouda // Journal of Failure Analysis and Prevention. - 2017. - Vol. 17, iss. 2. - P. 304-314. - DOI: 10.1007/s11668-017-0243-1.

12. Numerical and experimental validation of computational models for mode I composite fracture failure / J. Bonhomme, A. Argüelles, J. Viña, I. Viña // Computational Materials Science. 2009. - Vol. 45. - P. 993-998. - DOI: 10.1016/j.commatsci.2009.01.005.

13. Simulation of mode I delamination propagation in multidirectional composites with R-curve effects using VCCT method / M. M. Shokrieh, H. Rajabpour-Shirazi, M. Heidari-Rarani, M. Haghpanahi // Computational Materials Science. - 2012. - Vol. 65. - P. 66-73. DOI: 10.1016/j.commatsci.2012.06.025.

14. Computationally optimized formulation for the simulation of composite materials and delamination failures / X. Martinez, F. Rastellini, S. Oller, F. Floresa, E. Oñate // Composites Part B: Engineering. - 2011. - Vol. 42, iss. 2. - P. 134-144. - DOI: 10.1016/j.compositesb.2010.09.013.

15. Simulation of fatigue delamination growth in composite laminates under mode I loading / Yu. V. Skvortsov, S. A. Chernyakin, S. V. Glushkov, S. N. Perov // Applied Mathematical Modelling. - 2016. - Vol. 40. - P. 7216-7224. - DOI: 10.1016/j.apm.2016.03.019.

16. Amiri-Rad A., Mashayekhi M., Van der Meer F. P. Cohesive zone and level set method for simulation of high cycle fatigue delamination in composite materials // Composite Structures. 2017. - Vol. 160. - P. 61-69. - DOI: 10.1016/j.compstruct.2016.10.041.

17. May M., Hallett S. R. An advanced model for initiation and propagation of damage under fatigue loading - part I: Model formulation // Composite Structures. - 2011. - Vol. 93. - P. 23402349. - DOI: 10.1016/j.compstruct.2011.03.022.

18. Smirnov S. V., Myasnikova M. V., Igumnov A. S. Determination of the local shear strength of a layered metal composite material with a ductile interlayer after thermocycling // Diagnostics, Resource and Mechanics of materials and structures. - 2016. - Iss. 4. - P. 46-56. DOI: $10.17804 / 2410-9908.2016 .4 .046-056$.

19. Качанов Л. М. Основы механики разрушения. - М. : Наука, 1974. - 312 с. 